\title{
Spectral representations of sum- and max-stable processes
}

\author{
Zakhar Kabluchko
}

Received: 3 September 2008 / Revised: 16 February 2009 /

Accepted: 12 March 2009 / Published online: 1 April 2009

(C) The Author(s) 2009. This article is published with open access at Springerlink.com

\begin{abstract}
To each max-stable process with $\alpha$-Fréchet margins, $\alpha \in(0,2)$, a symmetric $\alpha$-stable process can be associated in a natural way. Using this correspondence, we deduce known and new results on spectral representations of max-stable processes from their $\alpha$-stable counterparts. We investigate the connection between the ergodic properties of a stationary max-stable process and the recurrence properties of the non-singular flow generating its spectral representation. In particular, we show that a stationary max-stable process is ergodic iff the flow generating its spectral representation has vanishing positive recurrent component. We prove that a stationary max-stable process is ergodic (mixing) iff the associated $\mathrm{S} \alpha \mathrm{S}$ process is ergodic (mixing). We construct nonsingular flows generating the max-stable processes of Brown and Resnick.
\end{abstract}

Keywords Symmetric $\alpha$-stable processes • Max-stable processes • Spectral representations $\cdot$ Non-singular flows

AMS 2000 Subject Classifications $60 \mathrm{G} 70 \cdot 60 \mathrm{G} 52$

\section{Introduction}

Symmetric $\alpha$-stable ( $\mathrm{S} \alpha \mathrm{S})$ and max-stable processes form two natural and rich classes of stochastic processes. For both classes, important role is played by the notion of spectral representation. Fundamental results on the existence and uniqueness of the spectral representation are due to Bretagnolle et al. (1966),

\footnotetext{
Z. Kabluchko $(\bowtie)$

Institut für Mathematische Stochastik, Georg-August-Universität Göttingen,

Goldschmidtstraße 7, 37077 Göttingen, Germany

e-mail:kabluch@math.uni-goettingen.de
} 
Schreiber (1972), Hardin (1982), Rosinski (1995) in the case of $\mathrm{S} \alpha \mathrm{S}$ processes and to de Haan (1984) and de Haan and Pickands (1986) in the case of maxstable processes.

Although there is similarity between the $\mathrm{S} \alpha \mathrm{S}$ and max-stable theories, the methods which were used in the papers cited above are quite different. In the $\mathrm{S} \alpha \mathrm{S}$ case, fundamental role is played by the rigidity of $L^{\alpha}$-spaces, whereas de Haan (1984) and de Haan and Pickands (1986) use more explicit constructions. Thus, it is natural to ask whether it is possible to construct some sort of correspondence between $\mathrm{S} \alpha \mathrm{S}$ and max-stable processes which allows to obtain the max-stable results from their $\mathrm{S} \alpha \mathrm{S}$ counterparts more or less automatically. It was noted in Stoev and Taqqu (2005) that it is possible to associate to each $S \alpha S$ process with non-negative spectral representation a maxstable process with the same spectral representation. Further, it was shown there that the pointwise maximum of $n$ independent copies of a $S \alpha \mathrm{S}$ process converges in distribution, as $n \rightarrow \infty$ and after normalization, to the associated max-stable process. Vice versa, in a natural way it is possible to associate to each max-stable process with $\alpha$-Fréchet margins, $\alpha \in(0,2)$, a $\mathrm{S} \alpha \mathrm{S}$ process. We give a precise definition of this association in Section 2 and show that most properties of a max-stable process are shared by the associated $\mathrm{S} \alpha \mathrm{S}$ process. This will be used in Section 3 to deduce the fundamental theorems of de Haan (1984) and de Haan and Pickands (1986) from their $\mathrm{S} \alpha \mathrm{S}$ counterparts.

The uniqueness of the spectral representation allows to establish a link between stationary $\mathrm{S} \alpha \mathrm{S}$ and max-stable processes and nonsingular flows on measure spaces, see Hardin (1982), de Haan and Pickands (1986), Rosinski (1995). In the $S \alpha S$ case, it was shown that the ergodic properties of a stationary process are closely related to the recurrence properties of the generating flow. By Hopf's theorem, the state space of the generating flow can be decomposed into conservative (or recurrent) and dissipative (or transient) parts. It was shown in Rosinski (1995) that stationary $\mathrm{S} \alpha \mathrm{S}$ processes having only dissipative part in their spectral representation are mixed moving averages processes (and, consequently, mixing). The conservative part may be further decomposed into positive recurrent and null recurrent parts. $\mathrm{S} \alpha \mathrm{S}$ processes generated by a flow with non-vanishing positive recurrent part were shown to be non-ergodic in Samorodnitsky (2005), whereas processes generated by a null recurrent flow are ergodic and may be both mixing and non-mixing, see Rosinski and Samorodnitsky (1996), Gross and Robertson (1993). In Sections 4 and 5 we prove analogous results for max-stable processes. We also prove a max-stable counterpart of the $S \alpha S$ result of Surgailis et al. (1993) giving a criterion for two mixed moving averages processes to have the same law.

A class of natural examples of stationary max-stable processes is provided by Brown-Resnick processes, which were introduced in Brown and Resnick (1977) in a special case and in Kabluchko et al. (2009) in general form. In Section 6 we construct explicitly nonsingular flows generating BrownResnick processes. We give sufficient conditions for these flows to be positive recurrent or dissipative. We also construct $\mathrm{S} \alpha \mathrm{S}$ counterparts of Brown-Resnick processes. 
We use the following notational conventions. If it is necessary to distinguish between $\mathrm{S} \alpha \mathrm{S}$ and max-stable objects (random variables, vectors, spectral measures, etc.), the former will have a superscript + , whereas the latter will have a superscript $\vee$. Note, however, that the subscript + denotes nonnegativity. By $\stackrel{\mathcal{D}}{=}$ we denote the equality of finite-dimensional distributions; $P$ lim denotes convergence in probability and $\vee$ stands for the maximum or supremum. If applied to vectors, the maximum is taken componentwise. If $(\Omega, \mathcal{A}, \mu)$ is a measure space and $\alpha>0$, then $L^{\alpha}(\Omega, \mathcal{A}, \mu)$ denotes the space of measurable functions $f: \Omega \rightarrow \mathbb{R}$ such that $\|f\|_{\alpha}=\left(\int_{\Omega}|f|^{\alpha} d \mu\right)^{1 / \alpha}<\infty$. All measure spaces are supposed to be complete. Finally, $L_{+}^{\alpha}(\Omega, \mathcal{A}, \mu)$ denotes the set of non-negative functions from $L^{\alpha}(\Omega, \mathcal{A}, \mu)$.

\section{$2 \mathrm{~S} \alpha \mathrm{S}$ processes associated to max-stable processes}

We start by recalling necessary facts about multivariate $\mathrm{S} \alpha \mathrm{S}$ and max-stable distributions. For more information, we refer to Samorodnitsky and Taqqu (1994) and Chapter 5 of Resnick (1987). A random vector $X=\left(X_{i}\right)_{i=1}^{n}$ whose distribution is symmetric with respect to the origin is called symmetric $\alpha$-stable $(\mathrm{S} \alpha \mathrm{S}), \alpha \in(0,2)$, if for each $r \in \mathbb{N}$ the random vector $X^{(1)}+\ldots+X^{(r)}$ has the same distribution as $r^{1 / \alpha} X$, where $X^{(1)}, \ldots, X^{(r)}$ are independent copies of $X$. Equivalently, $X$ is $\mathrm{S} \alpha \mathrm{S}$ if there is a finite symmetric measure $\Gamma$ on the unit sphere $\mathbb{S}^{n}$ in $\mathbb{R}^{n}$ such that the characteristic function of $X$ satisfies

$$
\mathbb{E} \exp \left(i \sum_{j=1}^{n} u_{j} X_{j}\right)=\exp \left(-\int_{\mathbb{S}^{n}}\left|\sum_{j=1}^{n} a_{j} u_{j}\right|^{\alpha} d \Gamma(a)\right) .
$$

The measure $\Gamma$ is uniquely determined and is called the spectral measure of $X$. Note that we always exclude the Gaussian case $\alpha=2$.

A fundamental role is played by the notion of $\mathrm{S} \alpha \mathrm{S}$ stochastic integral. There are several possible definitions; we need a definition in terms of Poisson processes. Let $(\Omega, \mathcal{A}, \mu)$ be a $\sigma$-finite measure space. Let $\left\{\left(\omega_{k}, y_{k}\right)\right\}_{k=1}^{\infty}$ be an enumeration of points of a Poisson point process on $\Omega \times \mathbb{R}$ with intensity $\mu \times$ $d y /|y|^{\alpha+1}$. For $f \in L^{\alpha}(\Omega, \mathcal{A}, \mu)$ and for $\alpha \in(0,1)$ the $\mathrm{S} \alpha \mathrm{S}$ stochastic integral of $f$ is defined by

$$
\int_{\Omega}^{+} f d M_{\mu}^{\alpha}=b_{\alpha} \sum_{k=1}^{\infty} y_{k} f\left(\omega_{k}\right)
$$

where $b_{\alpha}>0$ is a normalizing constant. The symbol $M_{\mu}^{\alpha}$ is to be understood as a random $S \alpha S$ measure with control measure $\mu$. The above definition does not work for $\alpha \geq 1$ since then the sum on the right-hand side of Eq. 2 
diverges. This difficulty can be overcome by introducing regularizing terms, see Samorodnitsky and Taqqu (1994).

If $f_{1}, \ldots, f_{n} \in L^{\alpha}(\Omega, \mathcal{A}, \mu)$, then, by Samorodnitsky and Taqqu (1994), the distribution of the vector $\left(\int_{\Omega}^{+} f_{j} d M_{\mu}^{\alpha}\right)_{j=1}^{n}$ is $\mathrm{S} \alpha \mathrm{S}$ and

$$
\mathbb{E} \exp \left(i \sum_{j=1}^{n}\left(u_{j} \int_{\Omega}^{+} f_{j} d M_{\mu}^{\alpha}\right)\right)=\exp \left(-\int_{\Omega}\left|\sum_{j=1}^{n} f_{j}(\omega) u_{j}\right|^{\alpha} d \mu(\omega)\right) .
$$

Now, we proceed to the max-stable case. In this paper, a random vector $X=$ $\left(X_{i}\right)_{i=1}^{n}$ is called max-stable if for every $r \in \mathbb{N}$ the componentwise maximum $X^{(1)} \vee \ldots \vee X^{(r)}$ has the same distribution as $r^{1 / \alpha} X$, where $X^{(1)}, \ldots, X^{(r)}$ are independent copies of $X$. Here, the parameter $\alpha$ takes values in $(0, \infty)$. We always suppose that the margins of $X$ are non-degenerate, in which case they are $\alpha$-Fréchet, that is they have distribution functions of the form $F_{c}^{\alpha}(t)=$ $\exp \left(-(c / t)^{\alpha}\right) 1_{t>0}, c>0$. A random vector $X$ is max-stable iff its distribution function satisfies

$$
\mathbb{P}\left[X_{1} \leq u_{1}, \ldots, X_{n} \leq u_{n}\right]=\exp \left(-\int_{\mathbb{S}_{+}^{n}} \vee_{i=1}^{n}\left(\frac{a_{i}}{u_{i}}\right)^{\alpha} d \Gamma(a)\right)
$$

for all $\left(u_{1}, \ldots, u_{n}\right) \in[0, \infty)^{n} \backslash\{0\}$, where $\Gamma$ is a finite measure on $\mathbb{S}_{+}^{n}=\mathbb{S}^{n} \cap$ $[0, \infty)^{n}$. Here, $0 / 0$ is interpreted as 0 . The measure $\Gamma$ is determined uniquely.

We recall the definition of max-stable stochastic integral introduced by de Haan (1984). Let $(\Omega, \mathcal{A}, \mu)$ be a $\sigma$-finite measure space. Let $\left\{\left(\omega_{k}, y_{k}\right)\right\}_{k=1}^{\infty}$ be an enumeration of points of a Poisson point process on $\Omega \times \mathbb{R}_{+}$with intensity $\mu \times d y / y^{\alpha+1}$. Let $\alpha \in(0, \infty)$. Then de Haan's stochastic integral of a function $f \in L_{+}^{\alpha}(\Omega, \mathcal{A}, \mu)$ is defined by

$$
\int_{\Omega}^{\vee} f d M_{\mu}^{\alpha}=c_{\alpha} \vee_{k=1}^{\infty} y_{k} f\left(\omega_{k}\right)
$$

where $c_{\alpha}=\alpha^{-1 / \alpha}$ is needed for normalization. If $f_{1}, \ldots, f_{n} \in L_{+}^{\alpha}(\Omega, \mathcal{A}, \mu)$, then the vector $\left(\int_{\Omega}^{\vee} f_{j} d M_{\mu}^{\alpha}\right)_{j=1}^{n}$ is max-stable and, by de Haan (1984), its distribution function is given by

$$
\mathbb{P}\left[\int_{\Omega}^{\vee} f_{i} d M_{\mu}^{\alpha} \leq u_{i} \forall i=1, \ldots, n\right]=\exp \left(-\int_{\Omega} \vee_{i=1}^{n}\left(\frac{f_{i}(\omega)}{u_{i}}\right)^{\alpha} d \mu(\omega)\right)
$$

for all $\left(u_{1}, \ldots, u_{n}\right) \in[0, \infty)^{n} \backslash\{0\}$.

Now we are ready to associate to each max-stable vector with $\alpha \in(0,2)$ a $\mathrm{S} \alpha \mathrm{S}$ vector. For a finite measure $\Gamma$ on the unit sphere $\mathbb{S}^{n}$ define its symmetrization $\Gamma^{\text {sym }}$ by $\Gamma^{\text {sym }}(A)=(\Gamma(A)+\Gamma(-A)) / 2$ for each Borel set $A \subset \mathbb{S}^{n}$.

Definition 1 Let $\alpha \in(0,2)$. A max-stable random vector $X^{\vee}$ (having $\alpha$-Fréchet margins) with spectral measure $\Gamma^{\vee}$ and a $\mathrm{S} \alpha \mathrm{S}$ vector $X^{+}$with spectral measure $\Gamma^{+}$are called associated if $\Gamma^{+}=\Gamma^{\vee s y m}$. 
The above definition applies to max-stable vectors with $\alpha \in(0,2)$ only (although, for general max-stable vectors, $\alpha$ takes values in $(0, \infty))$. To overcome this difficulty, we will use the following remark which shows that it possible to switch between max-stable vectors with different $\alpha$ 's using simple transformations. In many situations, it will allow us to assume without loss of generality that $\alpha=1$.

Remark 1 If $X^{\vee}=\left(X_{i}^{\vee}\right)_{i=1}^{n}$ is a max-stable vector with $\alpha$-Fréchet margins, $\alpha>0$, then for $\beta>0$, the vector $\left(X^{\vee}\right)^{\beta}=\left(\left(X_{i}^{\vee}\right)^{\beta}\right)_{i=1}^{n}$ is max-stable with $\alpha / \beta$ Fréchet margins.

Let us also stress that every max-stable vector with $\alpha \in(0,2)$ is associated to some $\mathrm{S} \alpha \mathrm{S}$ vector, but not every $\mathrm{S} \alpha \mathrm{S}$ vector can be associated to a max-stable one. More precisely, an $n$-dimensional $\mathrm{S} \alpha \mathrm{S}$ random vector is associated to some max-stable vector iff its spectral measure is concentrated on $\mathbb{S}_{+}^{n} \cup\left(-\mathbb{S}_{+}^{n}\right)$.

The next proposition extends the notion of association from random vectors to random processes. A stochastic process is called $\mathrm{S} \alpha \mathrm{S}$, resp. max-stable, if all of its finite-dimensional distributions are $\mathrm{S} \alpha \mathrm{S}$, resp. max-stable.

Proposition 1 Let $\left\{\eta^{\vee}(t), t \in T\right\}$ be a max-stable process with $\alpha \in(0,2)$. Then there is a $\alpha \alpha$ process $\left\{\eta^{+}(t), t \in T\right\}$ with the following property: for every $n \in \mathbb{N}$ and $t_{1}, \ldots, t_{n} \in T$, the $S \alpha S$ vector $\left(\eta^{+}\left(t_{i}\right)\right)_{i=1}^{n}$ and the max-stable vector $\left(\eta^{\vee}\left(t_{i}\right)\right)_{i=1}^{n}$ are associated.

To prove the above proposition we need to introduce some notation. If $a=$ $\left(a_{1}, \ldots, a_{n}\right)$ is a vector in $\mathbb{R}^{n}$, and $m \leq n$, we define $\sigma_{n, m} a=\left(a_{1}, \ldots, a_{m}\right)$. If $\Gamma$ is a finite measure on $\mathbb{R}^{n} \backslash\{0\}$, and $m \leq n$, we define a measure $\pi_{n, m} \Gamma$ on $\mathbb{S}^{m}$ as follows: for a Borel set $B \subset \mathbb{S}^{m}$ we let

$$
\left(\pi_{n, m} \Gamma\right)(B)=\int_{\text {cone }(B)}\left\|\sigma_{n, m} a\right\|_{2}^{\alpha} d \Gamma(a),
$$

where $\|\cdot\|_{2}$ denotes the Euclidian norm, and

$$
\operatorname{cone}(B)=\left\{a=\left(a_{1}, \ldots, a_{n}\right) \in \mathbb{S}^{n}: \sigma_{n, m} a /\left\|\sigma_{n, m} a\right\|_{2} \in B\right\} .
$$

It follows that for every non-negative Borel function $f$ on $\mathbb{S}^{m}$,

$$
\int_{\mathbb{S}^{m}} f(b) d\left(\pi_{n, m} \Gamma\right)(b)=\int_{\mathbb{S}^{n}} f\left(\frac{\sigma_{n, m} a}{\left\|\sigma_{n, m} a\right\|_{2}}\right)\left\|\sigma_{n, m} a\right\|_{2}^{\alpha} d \Gamma(a) .
$$

Note that if $\Gamma$ is symmetric, then so is $\pi_{n, m} \Gamma$; further, if $\Gamma$ is concentrated on $\mathbb{S}_{+}^{n}$, then $\pi_{n, m} \Gamma$ is concentrated on $\mathbb{S}_{+}^{m}$.

Lemma 1 Let $X=\left(X_{i}\right)_{i=1}^{n}$ be a max-stable, resp. S $\alpha S$, vector with spectral measure $\Gamma$ and let $m \leq n$. Then the vector $\left(X_{i}\right)_{i=1}^{m}$ is max-stable, resp. S $\alpha S$, and its spectral measure is $\pi_{n, m} \Gamma$. 
Proof We consider only the max-stable case. We compute the distribution function of the vector $\left(X_{i}\right)_{i=1}^{m}$. Fix $u_{1}, \ldots, u_{m}>0$, and let

$$
f\left(b_{1}, \ldots, b_{m}\right)=\vee_{i=1}^{m}\left(\frac{b_{i}}{u_{i}}\right)^{\alpha}, \quad b_{i} \geq 0 .
$$

Since $\left(X_{i}\right)_{i=1}^{n}$ is max-stable with spectral measure $\Gamma$, its distribution function is given by Eq. 4. Hence,

$$
\begin{aligned}
\mathbb{P}\left[X_{1} \leq u_{1}, \ldots, X_{m} \leq u_{m}\right] & =\mathbb{P}\left[\left(X_{1}, \ldots, X_{n}\right) \leq\left(u_{1}, \ldots, u_{m}, \infty, \ldots, \infty\right)\right] \\
& =\exp \left(-\int_{\mathbb{S}_{+}^{n}} \vee_{i=1}^{m}\left(\frac{a_{i}}{u_{i}}\right)^{\alpha} d \Gamma(a)\right) \\
& =\exp \left(-\int_{\mathbb{S}_{+}^{n}} f\left(\sigma_{n, m} a\right) d \Gamma(a)\right) \\
& =\exp \left(-\int_{\mathbb{S}_{+}^{n}} f\left(\frac{\sigma_{n, m} a}{\left\|\sigma_{n, m} a\right\|_{2}}\right)\left\|\sigma_{n, m} a\right\|_{2}^{\alpha} d \Gamma(a)\right) .
\end{aligned}
$$

Applying Eq. 7 and recalling that $\Gamma$ is concentrated on $\mathbb{S}_{+}^{n}$, we obtain

$$
\begin{aligned}
\mathbb{P}\left[X_{1} \leq u_{1}, \ldots, X_{m} \leq u_{m}\right] & =\exp \left(-\int_{\mathbb{S}_{+}^{m}} f(b) d\left(\pi_{n, m} \Gamma\right)(b)\right) \\
& =\exp \left(-\int_{\mathbb{S}_{+}^{m}} \vee_{i=1}^{m}\left(\frac{b_{i}}{u_{i}}\right)^{\alpha} d\left(\pi_{n, m} \Gamma\right)(b)\right) .
\end{aligned}
$$

Thus, the spectral measure of $\left(X_{i}\right)_{i=1}^{m}$ is $\pi_{n, m} \Gamma$. The $\mathrm{S} \alpha \mathrm{S}$ case can be treated analogously, by replacing Eq. 4 with Eq. 1 and considering characteristic functions instead of distribution functions.

Proof (Proof of Proposition 1) For $t_{1}, \ldots, t_{n} \in T$, let $\Gamma_{t_{1}, \ldots, t_{n}}^{\vee}$ be the spectral measure of the max-stable vector $\left(\eta^{\vee}\left(t_{i}\right)\right)_{i=1}^{n}$. Further, let $v_{t_{1}, \ldots, t_{n}}^{\vee}$ be the distribution of $\left(\eta^{\vee}\left(t_{i}\right)\right)_{i=1}^{n}$ and let $v_{t_{1}, \ldots, t_{n}}^{+}$be the $\mathrm{S} \alpha \mathrm{S}$ distribution associated to it, that is a $\mathrm{S} \alpha \mathrm{S}$ distribution with spectral measure $\Gamma_{t_{1}, \ldots, t_{n}}^{+}=\left(\Gamma_{t_{1}, \ldots, t_{n}}^{\vee}\right)^{\text {sym }}$.

We show that the family $\left\{v_{t_{1}, \ldots, t_{n}}^{+}: t_{1}, \ldots, t_{n} \in T, n \in \mathbb{N}\right\}$ is a consistent family of distributions and then apply Kolmogorov's extension theorem. Thus, we have to show that for $m \leq n$, the distribution of the first $m$ components of a $v_{t_{1}, \ldots, t_{n}}^{+}$-distributed random vector coincides with $v_{t_{1}, \ldots, t_{m}}^{+}$. Now, by the $\mathrm{S} \alpha \mathrm{S}$ part of Lemma 1, the spectral measure of the first $m$ components of a $v_{t_{1}, \ldots, t_{n}}^{+}$ distributed vector is $\pi_{n, m} \Gamma_{t_{1}, \ldots, t_{n}}^{+}$. We have

$$
\pi_{n, m} \Gamma_{t_{1}, \ldots, t_{n}}^{+}=\pi_{n, m}\left(\Gamma_{t_{1}, \ldots, t_{n}}^{\vee s y m}\right)=\left(\pi_{n, m} \Gamma_{t_{1}, \ldots, t_{n}}^{\vee}\right)^{s y m}=\left(\Gamma_{t_{1}, \ldots, t_{m}}^{\vee}\right)^{s y m}=\Gamma_{t_{1}, \ldots, t_{m}}^{+} .
$$

(The third equality follows from the max-stable part of Lemma 1). Thus, $\left\{v_{t_{1}, \ldots, t_{n}}^{+}\right\}$is a consistent family of distributions and we complete the proof by applying Kolmogorov's extension theorem. 
Remark 2 Above, we described how to associate to each max-stable process with $\alpha \in(0,2)$ a symmetric $\alpha$-stable process. Similarly, one can also associate to each max-stable process with $\alpha \in(0,2), \alpha \neq 1$, a totally skewed $\alpha$-stable process (in this case, no symmetrization in Definition 1 is needed). However, since the theory of spectral representations is more developed for $\mathrm{S} \alpha \mathrm{S}$ processes than for totally skewed $\alpha$-stable processes (for the latter, see Rosinski 1994), we prefer to work with $\mathrm{S} \alpha \mathrm{S}$ processes.

The next two simple lemmas will be often needed in the sequel.

Lemma 2 Let $f_{1}, \ldots, f_{n} \in L_{+}^{\alpha}(\Omega, \mathcal{A}, \mu), \alpha \in(0,2)$. Then the random vectors $X^{+}=\left(\int_{\Omega}^{+} f_{j} d M_{\mu}^{\alpha}\right)_{j=1}^{n}$ and $X^{\vee}=\left(\int_{\Omega}^{\vee} f_{j} d M_{\mu}^{\alpha}\right)_{j=1}^{n}$ are associated.

Proof We may suppose that there is no $\omega \in \Omega$ with $f_{j}(\omega)=0$ for all $j=$ $1, \ldots, n$. Define a map $F: \Omega \rightarrow[0, \infty)^{d} \backslash\{0\}$ by $F(\omega)=\left(f_{1}(\omega), \ldots, f_{n}(\omega)\right)$. Define a measure $v$ on $[0, \infty)^{d} \backslash\{0\}$ as the push-forward of the measure $\mu$ under the mapping $F$. Using Eq. 6, the spectral measure of $X^{\vee}$ is easily seen to be $\pi_{n, n} v$. Analogously, using Eq. 3, the spectral measure of $X^{+}$is $\left(\pi_{n, n} v\right)^{s y m}$. Thus, $X^{+}$and $X^{\vee}$ are associated.

Lemma 3 Let $\left\{\eta^{\vee}(t), t \in T\right\}$ be a max-stable process and denote by $\eta^{+}$the $S \alpha S$ process associated to $\eta^{\vee}$. Let $t, t_{k} \in T$ for $k \in \mathbb{N}$. Then

$$
P \lim _{k \rightarrow \infty} \eta^{\vee}\left(t_{k}\right)=\eta^{\vee}(t) \text { iff } P \lim _{k \rightarrow \infty} \eta^{+}\left(t_{k}\right)=\eta^{+}(t) .
$$

Proof Denote by $\Gamma_{k}^{\vee}$, resp. $\Gamma_{k}^{+}$, the spectral measure of the bivariate max-stable vector $X_{k}^{\vee}=\left(\eta^{\vee}\left(t_{k}\right), \eta^{\vee}(t)\right)$, resp. the bivariate $\mathrm{S} \alpha \mathrm{S}$ vector $X_{k}^{+}=$ $\left(\eta^{+}\left(t_{k}\right), \eta^{+}(t)\right)$. Then $\Gamma_{k}^{+}=\Gamma_{k}^{\vee s y m}$. We will show that

$$
P \lim _{k \rightarrow \infty} \eta^{\vee}\left(t_{k}\right)=\eta^{\vee}(t)
$$

iff the sequence of measures $\Gamma_{k}^{\vee}$ converges weakly to a measure concentrated on $D=\left\{\left(2^{-1 / 2}, 2^{-1 / 2}\right)\right\}$. An analogous statement, with a similar proof, holds in the $\mathrm{S} \alpha \mathrm{S}$ case, the limiting measure being concentrated on $D \cup-D$, and the statement of the lemma follows.

First, suppose that $\Gamma_{k}^{\vee}$ converges weakly to a measure $\Gamma^{\vee}$ concentrated on $D$. It follows from Eq. 4 that for every $u_{1}, u_{2}>0$,

$$
\begin{aligned}
\lim _{k \rightarrow \infty} \mathbb{P}\left[\eta^{\vee}\left(t_{k}\right) \leq u_{1}, \eta^{\vee}(t) \leq u_{2}\right] & =\lim _{k \rightarrow \infty} \exp \left(-\int_{\mathbb{S}_{+}^{2}} \vee_{i=1}^{2}\left(\frac{a_{i}}{u_{i}}\right)^{\alpha} d \Gamma_{k}^{\vee}\left(a_{1}, a_{2}\right)\right) \\
& =\exp \left(-\int_{\mathbb{S}_{+}^{2}} \vee_{i=1}^{2}\left(\frac{a_{i}}{u_{i}}\right)^{\alpha} d \Gamma^{\vee}\left(a_{1}, a_{2}\right)\right) \\
& =\exp \left(- \text { const } \cdot\left(\frac{1}{u_{1}^{\alpha}} \vee \frac{1}{u_{2}^{\alpha}}\right)\right) .
\end{aligned}
$$


The latter is the cumulative distribution function of a bivariate max-stable vector $X^{\vee}$ with a.s. identical components. We will show that this implies Eq. 8. For $\varepsilon>0$, set $\Delta_{\varepsilon}=\left\{(x, y) \in \mathbb{R}^{2}:|x-y|<\varepsilon\right\}$. By the portmanteau theorem, see e.g. (Billingsley 1999, p. 16), we have

$$
\limsup _{k \rightarrow \infty} \mathbb{P}\left[X_{k}^{\vee} \notin \Delta_{\varepsilon}\right] \leq \mathbb{P}\left[X^{\vee} \notin \Delta_{\varepsilon}\right]=0 .
$$

This implies Eq. 8.

Conversely, suppose that the sequence of measures $\Gamma_{k}^{\vee}$ has a limit point $\Gamma^{\vee}$ which is not concentrated on $D$. Then the sequence of distributions of $X_{k}^{\vee}$ has as limit point a distribution of some bivariate max-stable vector $X^{\vee}$ whose components are non-equal. Again by the portmanteau theorem, for some $\varepsilon>0$,

$$
\liminf _{k \rightarrow \infty} \mathbb{P}\left[X_{k}^{\vee} \notin \bar{\Delta}_{\varepsilon}\right] \geq \mathbb{P}\left[X^{\vee} \notin \bar{\Delta}_{\varepsilon}\right]>0,
$$

where $\bar{\Delta}_{\varepsilon}$ is the closure of $\Delta_{\varepsilon}$. This shows that Eq. 8 does not hold.

\section{Spectral representations of max-stable processes}

The notion of spectral representation plays a fundamental role in the theory of $\mathrm{S} \alpha \mathrm{S}$ and max-stable processes. In this section, we deduce the results of de Haan (1984) and de Haan and Pickands (1986) on the existence and uniqueness of the spectral representation of max-stable processes from their $\mathrm{S} \alpha \mathrm{S}$ counterparts.

Definition 2 A spectral representation of a $\mathrm{S} \alpha \mathrm{S}$ process $\left\{\eta^{+}(t), t \in T\right\}$ is a collection of functions $\left\{f_{t}\right\}_{t \in T} \subset L^{\alpha}(\Omega, \mathcal{A}, \mu)$, defined on some measure space $(\Omega, \mathcal{A}, \mu)$, such that

$$
\left\{\eta^{+}(t), t \in T\right\} \stackrel{\mathcal{D}}{=}\left\{\int_{\Omega}^{+} f_{t} d M_{\mu}^{\alpha}, t \in T\right\} .
$$

Similarly, a collection of functions $\left\{f_{t}\right\}_{t \in T} \subset L_{+}^{\alpha}(\Omega, \mathcal{A}, \mu)$ is called a spectral representation of a max-stable process $\left\{\eta^{\vee}(t), t \in T\right\}$ (with $\alpha$-Fréchet margins) if

$$
\left\{\eta^{\vee}(t), t \in T\right\} \stackrel{\mathcal{D}}{=}\left\{\int_{\Omega}^{\vee} f_{t} d M_{\mu}^{\alpha}, t \in T\right\} .
$$

The next lemma, complementing Remark 1, will allow us to assume without loss of generality that $\alpha \in(0,2)$ (or even $\alpha=1$ ) in considering spectral representations of max-stable processes.

Lemma 4 If $\left\{f_{t}\right\}_{t \in T} \subset L_{+}^{\alpha}(\Omega, \mathcal{A}, \mu)$ is a spectral representation of a max-stable process $\left\{\eta^{\vee}(t), t \in T\right\}$ with $\alpha$-Fréchet margins, and $\beta>0$, then $\left\{f_{t}^{\beta}\right\}_{t \in T} \subset$ 
$L_{+}^{\alpha / \beta}(\Omega, \mathcal{A}, \mu)$ is a spectral representation of the max-stable process $\left(\eta^{\vee}\right)^{\beta}$ having $\alpha / \beta$-Fréchet margins.

Proof Take $t_{1}, \ldots, t_{n} \in T$ and $\left(u_{1}, \ldots, u_{n}\right) \in[0, \infty)^{n} \backslash\{0\}$. Then the fact that $\left\{f_{t}\right\}_{t \in T}$ is a spectral representation of $\eta^{\vee}$, and Eq. 6 yield

$$
\begin{aligned}
\mathbb{P}\left[\left(\eta^{\vee}\left(t_{i}\right)\right)^{\beta} \leq u_{i} \forall i=1, \ldots, n\right] & =\mathbb{P}\left[\eta^{\vee}\left(t_{i}\right) \leq u_{i}^{1 / \beta} \forall i=1, \ldots, n\right] \\
& =\mathbb{P}\left[\int_{\Omega}^{\vee} f_{t_{i}} d M_{\mu}^{\alpha} \leq u_{i}^{1 / \beta} \forall i=1, \ldots, n\right] \\
& =\exp \left(-\int_{\Omega} \vee_{i=1}^{n}\left(\frac{f_{t_{i}}(\omega)}{u_{i}^{1 / \beta}}\right)^{\alpha} d \mu(\omega)\right) .
\end{aligned}
$$

Using trivial transformations and Eq. 6, we obtain

$$
\begin{aligned}
\mathbb{P}\left[\left(\eta^{\vee}\left(t_{i}\right)\right)^{\beta} \leq u_{i} \forall i=1, \ldots, n\right] & =\exp \left(-\int_{\Omega} \vee_{i=1}^{n}\left(\frac{f_{t_{i}}(\omega)^{\beta}}{u_{i}}\right)^{\alpha / \beta} d \mu(\omega)\right) \\
& =\mathbb{P}\left[\int_{\Omega}^{\vee} f_{t_{i}}^{\beta} d M_{\mu}^{\alpha / \beta} \leq u_{i} \forall i=1, \ldots, n\right] .
\end{aligned}
$$

This shows that $\left\{f_{t}^{\beta}\right\}_{t \in T}$ is a spectral representation of the process $\left(\eta^{\vee}\right)^{\beta}$.

By a fundamental theorem of de Haan (1984), any max-stable process defined on a countable state space $T$ (as well as any stochastically continuous max-stable process on $\mathbb{R}$ ) has a spectral representation. We start by proving a somewhat more general version of this theorem.

Theorem 1 Any max-stable process $\left\{\eta^{\vee}(t), t \in T\right\}$ has a spectral representation on some sufficiently rich measure space.

Proof By Lemma 4, we may suppose that $\alpha \in(0,2)$. Let $\left\{\eta^{+}(t), t \in T\right\}$ be the $\mathrm{S} \alpha \mathrm{S}$ process associated to $\eta^{\vee}$ as in Proposition 1. By Bretagnolle et al. (1966) and Schreiber (1972), the process $\eta^{+}$has a spectral representation $\left\{f_{t}\right\}_{t \in T}$ on some measure space $(\Omega, \mathcal{A}, \mu)$. We show that $\left\{\left|f_{t}\right|\right\}_{t \in T}$ is a spectral representation of $\eta^{\vee}$. Take $t_{1}, \ldots, t_{n} \in T$. Then the set

$$
A=\left\{\omega \in \Omega: f_{t_{i}}(\omega) \geq 0 \forall i=1, \ldots, n\right\} \cup\left\{\omega \in \Omega: f_{t_{i}}(\omega) \leq 0 \forall i=1, \ldots, n\right\}
$$

has full $\mu$-measure. Indeed, otherwise the spectral measure of the random vector $\left(\eta^{+}\left(t_{1}\right), \ldots, \eta^{+}\left(t_{n}\right)\right)$ would not be concentrated on $\mathbb{S}_{+}^{n} \cup\left(-\mathbb{S}_{+}^{n}\right)$. We show that the collection $\left\{\left|f_{t}\right|\right\}_{t \in T}$ forms another spectral representation of $\eta^{+}$. To this end, we compute the characteristic function of $\left(\int_{\Omega}^{+}\left|f_{t_{j}}\right| d M_{\mu}^{\alpha}\right)_{j=1}^{n}$ : using 
Eq. 3 in the first and third equalities, and the fact that $\mu(\Omega \backslash A)=0$ in the second equality, we obtain

$$
\begin{aligned}
\mathbb{E} \exp \left(i \sum_{j=1}^{n} u_{j} \int_{\Omega}^{+}\left|f_{t_{j}}\right| d M_{\mu}^{\alpha}\right) & =\exp \left(-\int_{\Omega}\left|\sum_{j=1}^{n}\right| f_{t_{j}}(\omega)\left|u_{j}\right|^{\alpha} d \mu(\omega)\right) \\
& =\exp \left(-\int_{\Omega}\left|\sum_{j=1}^{n} f_{t_{j}}(\omega) u_{j}\right|^{\alpha} d \mu(\omega)\right) \\
& =\mathbb{E} \exp \left(i \sum_{j=1}^{n} u_{j} \int_{\Omega}^{+} f_{t_{j}} d M_{\mu}^{\alpha}\right) \\
& =\mathbb{E} \exp \left(i \sum_{j=1}^{n} u_{j} \eta^{+}\left(t_{j}\right)\right) .
\end{aligned}
$$

So, the collection $\left\{\left|f_{t}\right|\right\}_{t \in T}$ is a spectral representation of $\eta^{+}$and thus, by Lemma 2, it is also a spectral representation of $\eta^{\vee}$.

The spectral representation is generally non-unique. In Hardin (1982) the notion of minimal spectral representation was introduced and it was shown that every $\mathrm{S} \alpha \mathrm{S}$ process satisfying the so-called condition $\mathrm{S}$ has a minimal representation which is moreover unique in some natural sense. For maxstable processes, an analogous result was proved in de Haan and Pickands (1986) by a different method. We deduce the result of de Haan and Pickands (1986) from its $\mathrm{S} \alpha \mathrm{S}$ counterpart. First we recall some definitions.

Definition 3 A spectral representation $\left\{f_{t}\right\}_{t \in T}$, defined on a measure space $(\Omega, \mathcal{A}, \mu)$, of a $\mathrm{S} \alpha \mathrm{S}$ (or max-stable) process is called minimal if the following two conditions are satisfied:

1. there is no set $B$ with $\mu(B)>0$ such that for all $t \in T$ we have $f_{t}=0 \mu$-a.e. on $B$.

2. the $\sigma$-algebra generated by the extended-valued functions $f_{t} / f_{s}, s, t \in T$, coincides with $\mathcal{A}$.

A stochastic process $\{\eta(t), t \in T\}$ is said to be separable in probability (or to satisfy condition S) if there is a countable set $T_{0} \subset T$ such that for every $t \in T$ there is a sequence $\left\{t_{k}\right\}_{k=1}^{\infty} \subset T_{0}$ such that $\eta(t)=P \lim _{k \rightarrow \infty} \eta\left(t_{k}\right)$. For example, condition $\mathrm{S}$ is satisfied if $T$ is a separable metric space and the process $\eta$ is stochastically continuous. Recall, see e.g. de la Rue (1993), that a probability space is called a Lebesgue space if it is isomorphic, as a measure space, to an interval $[0, a], 0 \leq a \leq 1$, with the Lebesgue measure, extended by at most countable number of atoms with total mass $1-a$. 
Now we reprove the following result of de Haan and Pickands (1986) (see also Stoev and Taqqu (2005) for the slightly more general version given below).

Theorem 2 Any max-stable process $\left\{\eta^{\vee}(t), t \in T\right\}$ satisfying condition $S$ has a minimal spectral representation defined on a Lebesgue probability space. The minimal representation is unique in the following sense: if $\left\{f_{t}^{1}\right\}_{t \in T}$ and $\left\{f_{t}^{2}\right\}_{t \in T}$ are two minimal representations of $\eta^{\vee}$, defined on Lebesgue probability spaces $\left(S_{1}, \mathcal{B}_{1}, \mu_{1}\right)$ and $\left(S_{2}, \mathcal{B}_{2}, \mu_{2}\right)$, then there is a non-singular measurable bijection $\pi: S_{2} \rightarrow S_{1}$ and a function $h \in L_{+}^{\alpha}\left(S_{2}, \mathcal{B}_{2}, \mu_{2}\right), h>0$, such that for all $t \in T$,

$$
f_{t}^{2}=h \cdot\left(f_{t}^{1} \circ \pi\right) \quad \text { and } \quad \frac{d\left(\mu_{1} \circ \pi\right)}{d \mu_{2}}=h^{\alpha} \quad \text { a.e. on } S_{2} .
$$

Proof Again by Lemma 4, we may assume that $\alpha \in(0,2)$. Let $\eta^{+}$be the $\mathrm{S} \alpha \mathrm{S}$ process associated to $\eta^{\vee}$, see Proposition 1. It follows from Lemma 3 that $\eta^{+}$satisfies condition (S). By Theorem 1.1 of Hardin (1982), $\eta^{+}$has a minimal spectral representation $\left\{f_{t}\right\}_{t \in T}$ defined on a Lebesgue probability space $(\Omega, \mathcal{A}, \mu)$. As in the proof of Theorem $1,\left\{\left|f_{t}\right|\right\}_{t \in T}$ is a spectral representation of $\eta^{\vee}$. We show that it is minimal. Condition 1 of Definition 3 is satisfied trivially. To show that Condition 2 holds, recall from the proof of Theorem 1 that for each $s, t \in T$ the sign of $f_{t}$ coincides with the sign of $f_{s} \mu$-a.e. and, consequently, the $\sigma$-algebra generated by $\left|f_{t}\right| /\left|f_{s}\right|$ coincides with the $\sigma$-algebra generated by $f_{t} / f_{s}$. However, the latter is equal to $\mathcal{A}$ since $\left\{f_{t}\right\}_{t \in T}$ was chosen to be a minimal representation of $\eta^{+}$.

We prove the uniqueness part of the theorem. Suppose that $\left\{f_{t}^{1}\right\}_{t \in T}$ and $\left\{f_{t}^{2}\right\}_{t \in T}$ are two minimal spectral representations of $\eta^{\vee}$ as described in the statement of the theorem. Both can be also viewed as minimal representations of the $\mathrm{S} \alpha \mathrm{S}$ process $\eta^{+}$associated to $\eta^{\vee}$ (Lemma 2). Thus, by the uniqueness result of Hardin (1982), there are $\pi$ and $h$ satisfying Eq. 9.

Let us note that a minimal representation of an $\mathrm{S} \alpha \mathrm{S}$ process exists even if the process does not satisfy condition S, see Hardin (1982). However, the underlying measure space is not Lebesgue in this case, and uniqueness of the minimal representation holds in a weaker form. Analogous results for maxstable processes can be easily obtained by the above method.

The uniqueness of the minimal spectral representation was used in Hardin (1982) and Rosinski (1995) to obtain a characterization of stationary $\mathrm{S} \alpha \mathrm{S}$ processes in terms of non-singular flows on measure spaces. It was shown there that each stochastically continuous stationary $\mathrm{S} \alpha \mathrm{S}$ process can be generated by a non-singular measurable flow on a Lebesgue probability space, an integrable function on the same space and a \pm 1 -valued cocycle. Analogous result for max-stable processes was obtained in de Haan and Pickands (1986) (note, however, that in the max-stable case the cocycle is not needed). We are going to deduce the result of de Haan and Pickands (1986) from its $\mathrm{S} \alpha \mathrm{S}$ counterpart. Additionally, we show that the generating flow can be taken to be measurable, this will be needed in the sequel. 
First, we need to recall the necessary definitions, see Krengel (1985, $\S \S 1.1,1.2)$ for more information. A flow on a measure space $(\Omega, \mathcal{A}, \mu)$ is a one-parameter family $\left\{\tau_{t}\right\}_{t \in \mathbb{R}}$ of measurable transformations of $\Omega$ satisfying the group property $\tau_{0}=i d, \tau_{t+s}=\tau_{t} \circ \tau_{s}, t, s \in \mathbb{R}$. Note, in particular, that all $\tau_{t}$ are invertible. A flow $\left\{\tau_{t}\right\}_{t \in \mathbb{R}}$ is called non-singular if every $\tau_{t}$ is non-singular, that is if $\tau_{t} \circ \mu \ll \mu$ for all $t \in \mathbb{R}$, where $\ll$ means absolute continuity of measures. A flow is called measurable if the map $(\omega, t) \mapsto \tau_{t}(\omega)$ is measurable as a map from $(\Omega \times \mathbb{R}, \mathcal{A} \times \mathcal{B})$ to $(\Omega, \mathcal{A})$ (here, $\mathcal{B}$ is the Borel $\sigma$-algebra on $\mathbb{R})$. A nonsingular flow on $(\Omega, \mathcal{A}, \mu)$ defines a one-parameter group $\left\{U_{t}\right\}_{t \in \mathbb{R}}$ of positivity preserving $L^{\alpha}(\Omega, \mathcal{A}, \mu)$-isometries by

$$
U_{t} f=\left(\frac{d \mu \circ \tau_{t}}{d \mu}\right)^{1 / \alpha} f \circ \tau_{t}, \quad f \in L^{\alpha}(\Omega, \mathcal{A}, \mu) .
$$

Theorem 3 Let $\left\{\eta^{\vee}(t), t \in \mathbb{R}\right\}$ be a stationary stochastically continuous maxstable process. Then there is a non-singular measurable flow $\left\{\tau_{t}\right\}_{t \in \mathbb{R}}$, defined on a Lebesgue probability space $(\Omega, \mathcal{A}, \mu)$, and a function $f_{0} \in L_{+}^{\alpha}(\Omega, \mathcal{A}, \mu)$ such that the collection of functions $\left\{f_{t}\right\}_{t \in \mathbb{R}}$ defined by

$$
f_{t}=U_{t} f_{0}
$$

is a minimal spectral representation of $\eta^{\vee}$.

Proof By Lemma 4, we suppose that $\alpha \in(0,2)$. Let $\eta^{+}$be the S $\alpha \mathrm{S}$ process associated to $\eta^{\vee}$. It is also stochastically continuous by Lemma 3. By Hardin (1982) and Theorem 3.1 of Rosinski (1995), $\eta^{+}$is generated by a triple consisting of a measurable nonsingular flow $\left\{\tau_{t}\right\}_{t \in \mathbb{R}}$ on a Lebesgue probability space $(\Omega, \mathcal{A}, \mu)$, a function $g_{0} \in L^{\alpha}(\Omega, \mathcal{A}, \mu)$ and a \pm 1 -valued cocycle $a_{t}(\omega)$ (see Rosinski 1995, for a definition) such that $\left\{g_{t}\right\}_{t \in \mathbb{R}}$, where $g_{t}=a_{t} \cdot U_{t}\left(g_{0}\right)$, is a spectral representation of $\eta^{+}$. Here, $U_{t}$ is as in Eq. 10. Then for $f_{t}=\left|g_{t}\right|$, Eq. 11 holds. Further, as in the proof of Theorem $1,\left\{f_{t}\right\}$ is a spectral representation of $\eta^{\vee}$. This completes the proof.

\section{Hopf decomposition and mixed moving maxima}

Definition 4 We say that a max-stable process $\left\{\eta^{\vee}(t), t \in \mathbb{R}\right\}$ is generated by a non-singular measurable flow $\left\{\tau_{t}\right\}_{t \in \mathbb{R}}$ on a $\sigma$-finite measure space $(\Omega, \mathcal{A}, \mu)$ and a function $f_{0} \in L_{+}^{\alpha}(\Omega, \mathcal{A}, \mu)$ if

1. $\left\{U_{t} f_{0}\right\}_{t \in \mathbb{R}}$ is a spectral representation of $\eta^{\vee}$.

2. there is no set $B \in \mathcal{A}$ such that for each $t \in \mathbb{R}$ we have $U_{t} f_{0}=0$ a.e. on $B$.

Here, $U_{t}$ is an isometry of $L^{\alpha}(\Omega, \mathcal{A}, \mu)$ defined by Eq. 10 . By Theorem 3 , each stochastically continuous stationary max-stable process is generated by some flow. A measure space on which a non-singular measurable flow acts can be decomposed into the so-called dissipative and conservative parts (Hopf decomposition). Our goal in this section is to study the dissipative part of the Hopf decomposition. 
First, we recall some definitions, see Krengel (1985, §1.3). Let $\tau$ be a nonsingular invertible measurable transformation of a measure space $(\Omega, \mathcal{A}, \mu)$. A set $W \in \mathcal{A}$ is called wandering if the sets $\tau^{k} W, k \in \mathbb{Z}$, are disjoint. The transformation $\tau$ is called conservative if there is no wandering set of positive measure. There is a $\tau$-invariant decomposition $\Omega=C \cup D$, called the Hopf decomposition, such that the restriction of $\tau$ to $C$ is conservative and $D$ can be written as $\cup_{k \in \mathbb{Z}} \tau^{k} W$ for some wandering set $W$. Now, if $\left\{\tau_{t}\right\}_{t \in \mathbb{R}}$ is a measurable non-singular flow, then the Hopf decomposition of $\Omega$ with respect to $\tau_{t}$ does not depend on $t \neq 0$ modulo $\mu$ and is called the Hopf decomposition generated by the flow $\left\{\tau_{t}\right\}_{t \in \mathbb{R}}$, see Krengel (1968, Lemma 2.1). A measurable non-singular flow $\left\{\tau_{t}\right\}_{t \in \mathbb{R}}$ on a measure space $(\Omega, \mathcal{A}, \mu)$ is called conservative (resp. dissipative) if $C=\Omega \bmod \mu(\operatorname{resp} . D=\Omega \bmod \mu)$.

Theorem 4 Let $\left\{\eta^{\vee}(t), t \in \mathbb{R}\right\}$ be a stationary max-stable process generated by a conservative (resp. dissipative) measurable non-singular flow. Then in any other representation it is generated by conservative (resp. dissipative) flow.

Proof The theorem follows by applying Theorem 4.1 of Rosinski (1995) to the associated $\mathrm{S} \alpha \mathrm{S}$ process $\eta^{+}$.

Theorem 5 Every stationary stochastically continuous max-stable process $\left\{\eta^{\vee}(t), t \in \mathbb{R}\right\}$ can be written as $\eta^{\vee}=\eta_{\text {cons }}^{\vee} \vee \eta_{\text {diss }}^{\vee}$, where $\eta_{\text {cons }}^{\vee}$ and $\eta_{\text {diss }}^{\vee}$ are independent stationary max-stable processes generated by conservative, resp. dissipative, flows. This decomposition is unique in distribution.

Proof By Theorem $3 \eta^{\vee}$ is generated by a flow $\left\{\tau_{t}\right\}_{t \in \mathbb{R}}$ on a measure space $(\Omega, \mathcal{A}, \mu)$ and a function $f_{0}$. Let $f_{t}=U_{t} f_{0}$. Define

$$
\eta_{\text {cons }}^{\vee}(t)=\int_{C}^{\vee} f_{t} d M_{\mu_{\mid C}}^{\alpha} \quad \text { and } \quad \eta_{\text {diss }}^{\vee}(t)=\int_{D}^{\vee} f_{t} d M_{\mu_{\mid D}}^{\alpha} .
$$

Then $\eta^{\vee}=\eta_{\text {cons }}^{\vee} \vee \eta_{\text {diss }}^{\vee}$ is the decomposition having all required properties. The uniqueness part of the theorem follows by applying Theorem 4.3 of Rosinski (1995) to the associated $\mathrm{S} \alpha \mathrm{S}$ process $\eta^{+}$.

Now we are going to show that the class of stationary max-stable processes generated by dissipative flows coincides with the class of mixed moving maxima processes. In the next definition, $(\mathbb{R}, \mathcal{B}, \lambda)$ is the real line endowed with the Borel $\sigma$-algebra $\mathcal{B}$ and the Lebesgue measure $\lambda$.

Definition 5 A stationary max-stable process $\left\{\eta^{\vee}(t), t \in \mathbb{R}\right\}$ is called mixed moving maxima process if there is a $\sigma$-finite measure space $(W, \mathcal{W}, \rho)$ and a function $g \in L_{+}^{\alpha}(W \times \mathbb{R}, \mathcal{W} \times \mathcal{B}, \rho \times \lambda)$ such that

$$
\left\{\eta^{\vee}(t), t \in \mathbb{R}\right\} \stackrel{\mathcal{D}}{=}\left\{\int_{W \times \mathbb{R}}^{\vee} g(w, s-t) d M_{\rho \times \lambda}^{\alpha}(w, s), t \in \mathbb{R}\right\} .
$$


Theorem 6 A stationary max-stable process $\left\{\eta^{\vee}(t), t \in \mathbb{R}\right\}$ is generated by a dissipative flow iff it has a mixed moving maxima representation.

Proof Analogous to the proof of Theorem 4.4 in Rosinski (1995).

In the next theorem we give a criterion which allows to decide, whether two given mixed moving maxima processes are equal in law. We deduce it from the corresponding $\mathrm{S} \alpha \mathrm{S}$ result due to Surgailis et al. (1993). Let $\eta^{\vee}$ be a mixed moving maxima process as in Definition 5. By Fubini's theorem, the map $w \mapsto g(w, \cdot)$ is a $\rho$-a.e. well-defined map from $W$ to $L_{+}^{\alpha}(\mathbb{R})$. We define a finite measure $v$ on $S_{+}=\left\{f \in L_{+}^{\alpha}(\mathbb{R}):\|f\|_{\alpha}=1\right\}$, endowed with the $\sigma$-algebra of Borel sets, by

$$
v(A)=\int_{W} 1_{A}\left(\frac{g(w, \cdot)}{\|g(w, \cdot)\|_{\alpha}}\right)\|g(w, \cdot)\|_{\alpha}^{\alpha} d \rho(w) .
$$

For two functions $f_{1}, f_{2} \in L^{\alpha}(\mathbb{R})$ we write $f_{1} \sim f_{2}$ if $f_{1}$ is a translate of $f_{2}$, that is if there is an $s \in \mathbb{R}$ such that $f_{1}(\cdot)=f_{2}(\cdot-s)$ a.e. Let $S_{+} / \sim$ be the quotient space, endowed with the $\sigma$-algebra of $\sim$-invariant sets, and let $\pi: S_{+} \rightarrow S_{+} / \sim$ be the quotient map.

Theorem 7 Two mixed moving maxima processes $\eta_{1}^{\vee}$ and $\eta_{2}^{\vee}$ are equal in law iff the corresponding measures $v_{1} \circ \pi^{-1}$ and $\nu_{2} \circ \pi^{-1}$ on $S_{+} / \sim$ are equal.

Proof Let $\left(W^{i}, \mathcal{W}^{i}, \rho^{i}\right), i=1,2$, be two $\sigma$-finite measure spaces and let $g_{i} \in$ $L_{+}^{1}\left(W^{i} \times \mathbb{R}, \mathcal{W}^{i} \times \mathcal{B}, \rho^{i} \times \lambda\right), i=1,2$. Let $\eta_{i}^{\vee}, i=1,2$, be the corresponding mixed moving maxima processes, see Definition 5. Construct the measures $v_{1}, v_{2}$ as above. Suppose that the laws of $\eta_{1}^{\vee}$ and $\eta_{2}^{\vee}$ are equal. Then the laws of the associated $\mathrm{S} \alpha \mathrm{S}$ processes are equal and it follows from Surgailis et al. (1993) that $v_{1} \circ \pi^{-1}=v_{2} \circ \pi^{-1}$. This proves the "only if" part of the theorem. The "if" part is easy, see Surgailis et al. (1993).

\section{A characterization of ergodicity}

A natural question is how to characterize ergodicity and mixing of a stationary $\mathrm{S} \alpha \mathrm{S}$ (or max-stable) process in terms of its generating flow. Characterizations of ergodicity and mixing for stationary $\mathrm{S} \alpha \mathrm{S}$ processes were given in Cambanis et al. (1987), Gross (1994), Gross and Robertson (1993), Samorodnitsky (2005). In the last paper, ergodicity was characterized in terms of the positivenull decomposition of the conservative part (see below): a stationary $\mathrm{S} \alpha \mathrm{S}$ process generated by a nonsingular measurable flow is ergodic iff the flow has no positive recurrent component. For max-stable processes, the question was studied in Stoev (2008). In particular, Stoev (2008) gives an easy verifiable necessary and sufficient condition for mixing. However, conditions of his Theorem 3.2 characterizing the ergodicity are difficult to verify. In Theorem 8 below we prove a max-stable counterpart of the result of Samorodnitsky 
(2005). Furthermore, we shall see in Theorem 9 below that ergodicity (resp. mixing) of a max-stable process is equivalent to the ergodicity (resp. mixing) of the associated $S \alpha \mathrm{S}$ process. It seems that this fact cannot be proved by a simple argument using the notion of association. Thus, our arguments in this section differ significantly from that of the previous sections.

The conservative part $C$ of a non-singular measurable flow $\left\{\tau_{t}\right\}_{t \in \mathbb{R}}$ on $(\Omega, \mathcal{A}, \mu)$ can be decomposed into a positive recurrent and null recurrent parts $P$ and $N$ in a way similar to the decomposition of the set of recurrent states of a Markov chain into positive recurrent and null recurrent states. For an exact definition we refer to Krengel (1985, Theorem 4.6 on p.141) and Samorodnitsky (2005). Here, we need only the following two properties of the positive-null decomposition. First, on the positive part $P$, there is a finite $\tau$-invariant measure $v$ such that $v \sim \mu$ (on $P$ ). Second, if $f \in L^{1}(\Omega, \mathcal{A}, \mu$ ), then Krengel's stochastic ergodic theorem, see Krengel (1985, Theorem 4.9 on p.143), says that for every measurable set $B \subset N$ of finite measure and every $\varepsilon>0$,

$$
\lim _{T \rightarrow \infty} \mu\left\{\omega \in B: \frac{1}{T} \int_{0}^{T} U_{t} f(\omega) d t>\varepsilon\right\}=0,
$$

where $U_{t}$ is as in Eq. 10. The next theorem is a max-stable counterpart of Samorodnitsky (2005), although the proof is different.

Theorem 8 Let $\left\{\eta^{\vee}(t), t \in \mathbb{R}\right\}$ be a stationary max-stable process generated by a measurable non-singular flow $\left\{\tau_{t}\right\}_{t \in \mathbb{R}}$ and a function $f_{0}$. Then $\eta^{\vee}$ is ergodic if and only if the flow $\left\{\tau_{t}\right\}_{t \in \mathbb{R}}$ has no positive recurrent component.

Proof First suppose that the positive recurrent component $P$ is non-trivial. Define $U_{t}$ as in Eq. 10 and let $f_{t}=U_{t} f_{0}$. By changing a measure on $P$, we may suppose that $\mu$ is invariant on $P$ and that $\mu(P)=1$. Let $\varepsilon$ be so small that

$$
\mu\left\{\omega \in P: f_{0}(\omega)>0\right\}>2 \varepsilon
$$

(the left-hand side is positive by Condition 2 of Definition 4, and by the fact that $P$ is $\tau$-invariant). Define the stationary max-stable processes $\eta_{P}^{\vee}$ and $\eta_{N}^{\vee}$ by restricting the spectral representation of $\eta^{\vee}$ to the positive recurrent, resp. null recurrent, part. More precisely, set

$$
\eta_{P}^{\vee}(t)=\int_{P}^{\vee} f_{t} d M_{\mu_{\mid P}}^{\alpha} \quad \text { and } \quad \eta_{N}^{\vee}(t)=\int_{N}^{\vee} f_{t} d M_{\mu_{\mid N}}^{\alpha} .
$$

Then $\eta^{\vee}=\eta_{P}^{\vee} \vee \eta_{N}^{\vee}$. We will show later that for every $C>0$,

$$
\mathbb{P}\left[\liminf _{T \rightarrow \infty} \frac{1}{T} \int_{0}^{T} 1_{\left\{\eta_{P}^{\vee}(t)<C\right\}} d t>1-\varepsilon\right] \neq 1 .
$$


We claim that this implies that $\eta^{\vee}$ is non-ergodic. Indeed, if $\eta^{\vee}$ were ergodic, then Birkhoff's ergodic theorem would imply that with $\mathbb{P}$-probability 1 ,

$$
\liminf _{T \rightarrow \infty} \frac{1}{T} \int_{0}^{T} 1_{\left\{\eta_{P}^{\vee}(t)<C\right\}} d t \geq \lim _{T \rightarrow \infty} \frac{1}{T} \int_{0}^{T} 1_{\left\{\eta^{\vee}(t)<C\right\}} d t=\mathbb{P}\left[\eta^{\vee}(0)<C\right] .
$$

However, this contradicts Eq. 14 if $C$ is so large that $\mathbb{P}\left[\eta^{\vee}(0)<C\right]>1-\varepsilon$.

So, we concentrate on proving Eq. 14. It follows from Eq. 13 that we can find a $\delta>0$ such that $\mu(A)>\varepsilon$, where $A=\left\{\omega \in P: f_{0}(\omega)>\delta\right\}$. Since the restriction of $\tau_{t}$ to $P$ is measure preserving, we have, by Birkhoff's ergodic theorem,

$$
\lim _{T \rightarrow \infty} \frac{1}{T} \int_{0}^{T} 1_{\left\{\tau_{t}(\omega) \in A\right\}} d t=g(\omega) \quad \mu-\text { a.e. on } P
$$

for some function $g$ with $\int_{P} g d \mu=\mu(A)>\varepsilon$. It follows that there is a set $B \subset P$ of positive measure such that $g_{\mid B}>\varepsilon$.

Now, from the definition of the extremal stochastic integral, see Eq. 5, we have

$$
\eta_{P}^{\vee}(t)=c_{\alpha} \vee_{k \in \mathbb{N}} y_{k} f_{t}\left(\omega_{k}\right)
$$

where $\left\{\left(\omega_{k}, y_{k}\right)\right\}_{k=1}^{\infty}$ is an enumeration of the points of the Poisson point process on $P \times \mathbb{R}_{+}$with intensity $\mu_{\mid P} \times d y / y^{\alpha+1}$. Without loss of generality assume that $y_{1}$ is the largest of all $y_{k}$. We have

$$
\mathbb{P}\left[y_{1} \geq \frac{C}{c_{\alpha} \delta}, \omega_{1} \in B\right]>0 .
$$

If the event $\left\{y_{1} \geq C /\left(c_{\alpha} \delta\right)\right\}$ occurs, then for each $t$ with $\tau_{t}\left(\omega_{1}\right) \in A$ we have

$$
\eta_{P}^{\vee}(t) \geq c_{\alpha} y_{1} f_{t}\left(\omega_{1}\right)=c_{\alpha} y_{1} f_{0}\left(\tau_{t}\left(\omega_{1}\right)\right)>c_{\alpha} \frac{C}{c_{\alpha} \delta} \cdot \delta=C,
$$

and thus, if additionally the event $\left\{\omega_{1} \in B\right\}$ occurs,

$$
\limsup _{T \rightarrow \infty} \frac{1}{T} \int_{0}^{T} 1_{\left\{\eta_{P}^{\vee}(t)<C\right\}} d t \leq \lim _{T \rightarrow \infty} \frac{1}{T} \int_{0}^{T} 1_{\left\{\tau_{t}\left(\omega_{1}\right) \notin A\right\}} d t=1-g\left(\omega_{1}\right)<1-\varepsilon .
$$

This proves Eq. 14.

Now we prove the "if" part of the theorem. Suppose that $\eta^{\vee}$ is generated by a measurable non-singular flow with vanishing positive recurrent part. For simplicity we may suppose that $\alpha=1$. By Theorem 3.2 of Stoev (2008), in order to show that $\eta^{\vee}$ is ergodic it suffices to show that for every $f \in L_{+}^{1}(\Omega, \mathcal{A}, \mu)$,

$$
\lim _{T \rightarrow \infty} \frac{1}{T} \int_{0}^{T}\left\|f \wedge U_{t} f\right\|_{1} d t=0
$$


Here, $\wedge$ denotes the minimum. Choose a set $A_{1} \subset \Omega$ of finite measure such that $\int_{\Omega \backslash A_{1}} f \leq \varepsilon$. Further, let the constant $c$ be so large that $\int_{A_{2}} f d \mu<\varepsilon$ where $A_{2}=\left\{\omega \in A_{1}: f>c\right\}$. Set $A=A_{1} \backslash A_{2}$. Then

$$
\left\|f \wedge U_{t} f\right\|_{1} \leq \int_{\Omega \backslash A} f d \mu+\int_{A}\left(f \wedge U_{t} f\right) d \mu \leq 2 \varepsilon+\int_{A}\left(f \wedge U_{t} f\right) d \mu .
$$

By Krengel's stochastic ergodic theorem, see Eq. 12, and with the notation

$$
A_{3}(T)=\left\{\omega \in A: \frac{1}{T} \int_{0}^{T} U_{t} f(\omega) d t>\frac{\varepsilon}{\mu(A)}\right\},
$$

we have $\lim _{T \rightarrow \infty} \mu\left(A_{3}(T)\right)=0$. Thus, if $T$ is sufficiently large, then $\mu\left(A_{3}(T)\right)<\varepsilon / c$. We are going to show that Eq. 15 holds. If $T$ is large, then

$$
\begin{aligned}
\int_{0}^{T}\left\|f \wedge U_{t} f\right\|_{1} d t \leq & \int_{0}^{T}\left(2 \varepsilon+\int_{A}\left(f(\omega) \wedge U_{t} f(\omega)\right) d \mu(\omega)\right) d t \\
= & 2 T \varepsilon+\int_{A} \int_{0}^{T}\left(f(\omega) \wedge U_{t} f(\omega)\right) d t d \mu(\omega) \\
\leq & 2 T \varepsilon+\int_{A_{3}(T)} \int_{0}^{T} f(\omega) d t d \mu(\omega) \\
& +\int_{A \backslash A_{3}(T)} \int_{0}^{T}\left(f(\omega) \wedge U_{t} f(\omega)\right) d t d \mu(\omega) \\
\leq & 2 T \varepsilon+\int_{A_{3}(T)} c T d \mu(\omega)+\int_{A \backslash A_{3}(T)} \frac{T \varepsilon}{\mu(A)} d \mu(\omega) \\
\leq & 4 T \varepsilon .
\end{aligned}
$$

This, since $\varepsilon>0$ was arbitrary, proves Eq. 15 and the ergodicity of $\eta^{\vee}$.

Theorem 9 Let $\eta^{\vee}$ be a stationary max-stable process with $\alpha \in(0,2)$, generated by a measurable non-singular flow $\left\{\tau_{t}\right\}_{t \in \mathbb{R}}$ and a function $f_{0}$. Let $\eta^{+}$be the $S \alpha S$ process associated to $\eta^{\vee}$. Then $\eta^{\vee}$ is ergodic (resp. mixing) iff $\eta^{+}$is ergodic (resp. mixing).

Proof Let $f_{t}=U_{t} f_{0}$, where $U_{t}$ is as in Eq. 10. Then $\left\{f_{t}\right\}_{t \in \mathbb{R}}$ is a spectral representation for both $\eta^{\vee}$ and $\eta^{+}$. By Theorem $8, \eta^{\vee}$ is ergodic iff the positive recurrent component of the flow $\left\{\tau_{t}\right\}_{t \in \mathbb{R}}$ vanishes. Since by Theorem 3.1 of Samorodnitsky (2005) the same is true for $\eta^{+}$, the ergodic part of the theorem is established.

We prove the mixing part of the theorem. For simplicity we suppose that $\alpha=1$. By Theorem 3.3 of Stoev (2008), $\eta^{\vee}$ is mixing iff

$$
\lim _{t \rightarrow \infty}\left\|f_{0} \wedge f_{t}\right\|_{1}=0
$$


On the other hand, by Gross (1994), $\eta^{+}$is mixing iff for every compact set $K \subset(0, \infty)$ and $\varepsilon>0$

$$
\lim _{t \rightarrow \infty} \mu\left\{\omega: f_{0}(\omega) \in K, f_{t}(\omega)>\varepsilon\right\}=0 .
$$

We show that Eqs. 16 and 17 are equivalent. Suppose that Eq. 16 holds. Define $\delta=\min \left(\inf _{t \in K} t, \varepsilon\right)$. Then $\delta>0$ and we have

$$
\begin{aligned}
\mu\left\{\omega: f_{0}(\omega) \in K, f_{t}(\omega)>\varepsilon\right\} & \leq \mu\left\{\omega: f_{0}(\omega)>\delta, f_{t}(\omega)>\delta\right\} \\
& =\mu\left\{\omega:\left(f_{0} \wedge f_{t}\right)(\omega)>\delta\right\} \\
& \leq \delta^{-1}\left\|f_{0} \wedge f_{t}\right\|_{1} .
\end{aligned}
$$

Letting $t \rightarrow \infty$ and using Eq. 16, we obtain Eq. 17 .

Now suppose that Eq. 17 holds. Find a set $A$ of finite measure and a constant $c$ such that $\int_{\Omega \backslash A} f_{0} \leq \varepsilon$ and $f_{0}<c$ on $A$. Let

$$
A(t)=\left\{\omega \in A:\left(f_{0} \wedge f_{t}\right)(\omega)>\frac{\varepsilon}{\mu(A)}\right\} .
$$

By taking $K=[\varepsilon / \mu(A), c]$ and $\varepsilon / \mu(A)$ instead of $\varepsilon$ in Eq. 17, we obtain $\lim _{t \rightarrow \infty} \mu(A(t))=0$. Thus, for $t$ sufficiently large, we have $\mu(A(t)) \leq \varepsilon / c$. We obtain, for $t$ large,

$$
\begin{aligned}
\int_{\Omega}\left(f_{0} \wedge f_{t}\right) d \mu & =\int_{\Omega \backslash A}\left(f_{0} \wedge f_{t}\right) d \mu+\int_{A(t)}\left(f_{0} \wedge f_{t}\right) d \mu+\int_{A \backslash A(t)}\left(f_{0} \wedge f_{t}\right) d \mu \\
& \leq \varepsilon+c \mu(A(t))+\mu(A) \varepsilon / \mu(A) \\
& \leq 3 \varepsilon
\end{aligned}
$$

This proves Eq. 16.

Using the notion of association, it is straightforward to deduce from the $\mathrm{S} \alpha \mathrm{S}$ results of Samorodnitsky (2005) that a stationary max-stable process generated by a positive recurrent (resp. null recurrent) flow in some representation must be generated by positive recurrent (resp. null recurrent) flow in any other representation. Further, in the decomposition $\eta^{\vee}=\eta_{\text {cons }}^{\vee} \vee \eta_{\text {diss }}^{\vee}$ of a stationary stochastically continuous max-stable process $\eta^{\vee}$ (Theorem 5 above) the conservative component $\eta_{\text {cons }}^{\vee}$ can be represented as $\eta_{\text {cons }}^{\vee}=\eta_{P}^{\vee} \vee \eta_{N}^{\vee}$, where $\eta_{P}^{\vee}$ and $\eta_{N}^{\vee}$ are independent stationary max-stable processes generated by positive recurrent and null recurrent flow respectively. The positive recurrent component $\eta_{P}^{\vee}$ can be represented as

$$
\eta_{P}^{\vee}(t)=\vee_{k=1}^{\infty} y_{k} X_{k}(t), \quad t \in \mathbb{R},
$$

where $\left\{y_{k}\right\}_{k=1}^{\infty}$ is an enumeration of points of a Poisson point process on $\mathbb{R}_{+}$with intensity $d y / y^{\alpha+1}$ and $X_{k}, k \in \mathbb{N}$, are independent copies of some stationary non-negative-valued stochastic process $\{X(t), t \in \mathbb{R}\}$. Here is a short proof of this. After changing a measure on $P$, we may assume that $\left\{\tau_{t}\right\}_{t \in \mathbb{R}}$ is 
measure preserving on $P$ and that $\mu(P)=1$. By the definition of the extremal stochastic integral (5) we obtain

$$
\eta_{P}^{\vee}(t)=c_{\alpha} \vee_{k=1}^{\infty} y_{k} U_{t} f_{0}\left(\omega_{k}\right)
$$

where $\omega_{k}, k \in \mathbb{N}$, are elements from $P$, chosen independently according to the measure $\mu_{\mid P}$. To complete the proof, define independent stationary stochastic processes $\left\{X_{k}(t), t \in \mathbb{R}\right\}, k \in \mathbb{N}$, by $X_{k}(t)=c_{\alpha} U_{t} f_{0}\left(\omega_{k}\right)$. Thus, stationary maxstable processes generated by positive recurrent flows are exactly those considered in Theorem 2 of Schlather (2002). The null-recurrent component $\eta_{N}^{\vee}$ is less tractable.

\section{Spectral representations of Brown-Resnick processes}

In this section, we find an explicit construction of non-singular flows generating a class of stationary max-stable processes which will be called Brown-Resnick processes. These processes arise naturally as limits of pointwise maxima of large number of suitably normed and spatially rescaled Gaussian processes, see Brown and Resnick (1977) and Kabluchko et al. (2009). First, we recall the definition of these processes (note that the processes we are considering have Fréchet margins).

Let $W=\left\{W_{t}, t \in \mathbb{R}\right\}$ be a Gaussian process with stationary increments, defined on some probability space $(\Omega, \mathcal{A}, \mu)$. We always suppose that $W$ has zero mean and continuous sample paths. Recall that stationarity of increments means that the law of the process $\left\{W_{t+h}-W_{h}, t \in \mathbb{R}\right\}$ does not depend on $h \in \mathbb{R}$. Let $\sigma_{t}^{2}=\operatorname{Var} W_{t}$ be the variance and $\gamma_{t}=\mathbb{E}\left[\left(W_{t}-W_{0}\right)^{2}\right]$ the incremental variance (or variogram) of $W$. The covariance function of $W$ is given by

$$
\operatorname{Cov}\left(W_{t}, W_{s}\right)=\frac{1}{2}\left(\sigma_{t}^{2}+\sigma_{s}^{2}-\gamma_{t-s}\right)
$$

Let $\xi_{t}=W_{t}-\sigma_{t}^{2} / 2$. The max-stable process $\left\{\eta^{\vee}(t), t \in \mathbb{R}\right\}$ defined by

$$
\eta^{\vee}(t)=\int_{\Omega}^{\vee} \exp \left(\xi_{t}\right) d M_{\mu}^{1}
$$

will be called the Brown-Resnick process associated to $W$. It is clear that $\eta^{\vee}$ is max-stable with unit Fréchet margins. Using the definition of extremal stochastic integral (5), the above definition of $\eta^{\vee}$ can be reformulated as follows: Let $\left\{\xi_{t}^{(k)}, t \in \mathbb{R}\right\}, k \in \mathbb{N}$, be independent copies of $\left\{\xi_{t}, t \in \mathbb{R}\right\}$ and let $\left\{y_{k}\right\}_{k=1}^{\infty}$ be an enumeration of points of an independent Poisson point process on $\mathbb{R}_{+}$with intensity $d y / y^{2}$. Then

$$
\eta^{\vee}(t)=\vee_{k=1}^{\infty} y_{k} \exp \left(\xi_{t}^{(k)}\right)
$$

Although this is by no means evident from the spectral representation Eq. 18, Brown-Resnick processes turn out to be stationary. This was proved by Brown and Resnick (1977) for $W$ being a Brownian motion, and in Kabluchko et al. (2009) in the general case. 
The process $\eta^{\vee}$, being stationary, max-stable and continuous in probability, must be generated by a non-singular flow by Theorem 3. Our goal will be to construct this flow explicitly. To do this, we first define the probability space on which the flow acts. Let $C_{0}(\mathbb{R})$ be the space of continuous functions on $\mathbb{R}$ vanishing at zero. We endow $C_{0}(\mathbb{R})$ with the topology of uniform convergence on compact sets and denote the corresponding Borel $\sigma$-algebra by $\mathcal{B}$. Let $\tilde{W}=\left\{\tilde{W}_{t}, t \in \mathbb{R}\right\}$ be the process defined by $\tilde{W}_{t}=W_{t}-W_{0}$. Note that $\tilde{W}$ is a Gaussian process with stationary increments having the same variogram $\gamma_{t}$ as $W$. Note also that $\tilde{W}_{0}=0$ a.s. and $\operatorname{Var} \tilde{W}_{t}=\gamma_{t}$. Denote by $v$ the law of the drifted process $\tilde{\xi}_{t}=\tilde{W}_{t}-\gamma_{t} / 2$ on the space $C_{0}(\mathbb{R})$. Now we define a flow on the space $\left(C_{0}(\mathbb{R}), \mathcal{B}, v\right)$ as follows: for $t \in \mathbb{R}$ we define $\tau_{t}: C_{0}(\mathbb{R}) \rightarrow C_{0}(\mathbb{R})$ by

$$
\left(\tau_{t} \omega\right)(s)=\omega(s+t)-\omega(t), \quad \omega \in C_{0}(\mathbb{R}) .
$$

Theorem $10\left\{\tau_{t}\right\}_{t \in \mathbb{R}}$ is a measurable non-singular flow on the Lebesgue probability space $\left(C_{0}(\mathbb{R}), \mathcal{B}, v\right)$, which, together with $f_{0}=1$, generates a minimal spectral representation of the Brown-Resnick process $\eta^{\vee}$ in the sense of Definition 4.

The proof of Theorem 10 will be based on the following auxiliary result.

Proposition 2 For each $h \in \mathbb{R}$ the law of the process $\left\{\xi_{t+h}-\xi_{h}, t \in \mathbb{R}\right\}$ under the probability measure $e^{\xi_{h}} d \mu$ is the same as the law of $\left\{\tilde{\xi}_{t}, t \in \mathbb{R}\right\}$.

Proof First note that $\int_{\Omega} e^{\xi_{h}} d \mu=1$ and so $e^{\xi_{h}} d \mu$ is indeed a probability measure on $\Omega$. Take $t_{1}, \ldots, t_{n} \in \mathbb{R}$. The Laplace transform $\varphi$ of the random vector $\left(\xi_{i}+h-\xi_{h}\right)_{i=1}^{n}$ under $e^{\xi_{h}} d \mu$ is given by

$$
\begin{aligned}
\varphi\left(\alpha_{1}, \ldots, \alpha_{n}\right) & =\int_{\Omega} \exp \left(\alpha_{1}\left(\xi_{t_{1}+h}-\xi_{h}\right)+\ldots+\alpha_{n}\left(\xi_{t_{n}+h}-\xi_{h}\right)\right) e^{\xi_{h}} d \mu \\
& =\int_{\Omega} \exp \left(\alpha_{1} \xi_{t_{1}+h}+\ldots+\alpha_{n} \xi_{t_{n}+h}+\left(1-\alpha_{1}-\ldots-\alpha_{n}\right) \xi_{h}\right) d \mu .
\end{aligned}
$$

Now, under the probability measure $\mu$, the random vector $\left(\xi_{t_{1}+h}, \ldots, \xi_{t_{n}+h}, \xi_{h}\right)$ is Gaussian with easily computable expectation and covariance. A calculation similar to that in the proof of Theorem 10 in Kabluchko et al. (2009) shows that

$$
\varphi\left(\alpha_{1}, \ldots, \alpha_{n}\right)=\exp \left(-\frac{1}{2} \sum_{i=1}^{n} \gamma_{t_{i}} \alpha_{i}+\frac{1}{4} \sum_{i, j=1}^{n}\left(\gamma_{t_{i}}+\gamma_{t_{j}}-\gamma_{t_{i}-t_{j}}\right) \alpha_{i} \alpha_{j}\right),
$$

which is exactly the Laplace transform of the vector $\left(\tilde{\xi}_{t_{1}}, \ldots, \tilde{\xi}_{t_{n}}\right)$. This completes the proof.

As a byproduct we obtain a result already proved in Kabluchko et al. (2009).

Corollary 1 The process $\eta^{\vee}$ is stationary. Its law depends only on the variogram $\gamma_{t}$ of the underlying Gaussian process $W$. 
Proof Take $h, t_{1}, \ldots, t_{n} \in \mathbb{R}$. We have

$$
\left(\eta^{\vee}\left(t_{i}+h\right)\right)_{i=1}^{n} \stackrel{\mathcal{D}}{=}\left(\int_{\Omega}^{\vee} e^{\xi_{i}+h} d M_{\mu}^{1}\right)_{i=1}^{n} \stackrel{\mathcal{D}}{=}\left(\int_{\Omega}^{\vee} e^{\xi_{i}+h-\xi_{h}} d M_{e^{\xi_{h}} d \mu}^{1}\right)_{i=1}^{n} .
$$

Here, the second equality follows from Eq. 6. Applying to the right-hand side Proposition 2, we obtain

$$
\left(\eta^{\vee}\left(t_{i}+h\right)\right)_{i=1}^{n} \stackrel{\mathcal{D}}{=}\left(\int_{\Omega}^{\vee} e^{\tilde{\xi}_{i}} d M_{\mu}^{1}\right)_{i=1}^{n} .
$$

Thus, the distribution of $\left(\eta^{\vee}\left(t_{i}+h\right)\right)_{i=1}^{n}$ does not depend on $h$, which proves the stationarity of $\eta^{\vee}$. Further, the law of the process $\tilde{\xi}_{t}$ is completely determined by the variogram $\gamma_{t}$, which proves the second part of the corollary.

Proof (Proof of Theorem 10) Since $v$ is a Borel probability measure on the Polish space $C_{0}(\mathbb{R})$, it follows that $\left(C_{0}(\mathbb{R}), \mathcal{B}, v\right)$ is a Lebesgue space, see e.g. (de la Rue 1993, Theorem 2-3). We show that $\left\{\tau_{t}\right\}_{t \in \mathbb{R}}$ is a measurable nonsingular flow on $C_{0}(\mathbb{R})$. The measurability is clear. The group property is also easily verified:

$$
\begin{aligned}
\tau_{t_{1}}\left(\tau_{t_{2}} \omega(s)\right) & =\left.\tau_{t_{1}}\left(\omega\left(\cdot+t_{2}\right)-\omega\left(t_{2}\right)\right)\right|_{\cdot=s} \\
& =\left.\left(\omega\left(\cdot+t_{1}+t_{2}\right)-\omega\left(t_{2}\right)-\omega\left(t_{1}+t_{2}\right)+\omega\left(t_{2}\right)\right)\right|_{\cdot=s} \\
& =\omega\left(s+t_{1}+t_{2}\right)-\omega\left(t_{1}+t_{2}\right) \\
& =\tau_{t_{1}+t_{2}} \omega(s) .
\end{aligned}
$$

We show that for each $h \in \mathbb{R}$ the transformation $\tau_{h}$ is non-singular. More precisely, we claim that

$$
\frac{d\left(v \circ \tau_{h}\right)}{d v}(\omega)=e^{\omega(h)} .
$$

Let $B \subset C_{0}(\mathbb{R})$ be a $\mathcal{B}$-measurable set. It follows from Proposition 2 applied to $\tilde{W}$ that

$$
\int_{\Omega} 1_{\left(\tilde{\xi}_{t+h}(\cdot)-\tilde{\xi}_{h}(\cdot)\right) \in B} e^{\tilde{\xi}_{h}} d \mu=\int_{\Omega} 1_{\tilde{\xi}_{t}(\cdot) \in B} d \mu .
$$

This may be written as

$$
\int_{C_{0}(\mathbb{R})} 1_{\tau_{h} \omega \in B} e^{\omega(h)} d \nu(\omega)=\int_{C_{0}(\mathbb{R})} 1_{\omega \in B} d \nu(\omega) .
$$

It follows that

$$
\int_{\tau_{h}^{-1} B} e^{\omega(h)} d v(\omega)=v(B) .
$$

Writing in this equality $\tau_{h} B$ instead of $B$ we obtain

$$
\int_{B} e^{\omega(h)} d v(\omega)=v\left(\tau_{h}(B)\right) .
$$


This proves Eq. 20. Now we show that the flow $\left\{\tau_{t}\right\}_{t \in \mathbb{R}}$, together with the function $f_{0}=1$, generates a spectral representation of the Brown-Resnick process $\eta^{\vee}$ in the sense of Definition 4. Let $f_{t}=U_{t} f_{0}$. We have

$$
f_{t}(\omega)=\frac{d \nu \circ \tau_{t}}{d \nu}(\omega) f_{0}\left(\tau_{t}(\omega)\right)=\frac{d \nu \circ \tau_{t}}{d v}(\omega)=e^{\omega(t)} .
$$

Recall that $v$ is the law of the random process $\tilde{\xi}_{t}$. Thus

$$
\int_{C_{0}(\mathbb{R})}^{\vee} f_{t}(\omega) d M_{\nu}^{1}(\omega)=\int_{C_{0}(\mathbb{R})}^{\vee} e^{\omega(t)} d M_{\nu}^{1}(\omega)=\int_{\Omega}^{\vee} e^{\tilde{\xi}_{t}} d M_{\mu}^{1} .
$$

Applying to the right-hand side Eq. 19 with $h=0$, we obtain

$$
\left\{\int_{C_{0}(\mathbb{R})}^{\vee} f_{t}(\omega) d M_{\nu}^{1}(\omega), t \in \mathbb{R}\right\} \stackrel{\mathcal{D}}{=}\left\{\eta^{\vee}(t), t \in \mathbb{R}\right\} .
$$

This shows that $\left\{f_{t}\right\}_{t \in \mathbb{R}}$ is a spectral representation of $\eta^{\vee}$. Finally, we show that the representation $\left\{f_{t}\right\}_{t \in \mathbb{R}}$ is minimal. Clearly, the $\sigma$-algebra generated by $f_{t} / f_{s}, t, s \in \mathbb{R}$, is contained in $\mathcal{B}$. On the other hand, it contains the $\sigma$-algebra generated by $f_{t}, t \in \mathbb{R}$, (set $s=0$ and recall that $f_{0}=1$ ), which coincides with $\mathcal{B}$. This completes the proof.

The explicit construction of the spectral representation of the BrownResnick processes $\eta^{\vee}$ together with the results of the previous section allows to say more about the ergodic properties of $\eta^{\vee}$.

Theorem 11 Suppose that

$$
\lim _{t \rightarrow \infty}\left(W_{t}-\sigma_{t}^{2} / 2\right)=-\infty \text { a.s. }
$$

Then the corresponding process $\eta^{\vee}$ is generated by a dissipative flow.

Proof Let $\left\{\tau_{t}\right\}_{t \in \mathbb{R}}$ be the flow constructed in the proof of Theorem 10. By Krengel (1968, Lemma 2.1), the flow $\left\{\tau_{t}\right\}_{t \in \mathbb{R}}$ is dissipative iff $\tau_{1}$ is dissipative. To show that $\tau_{1}$ is dissipative it suffices to construct a countable covering $\left\{A_{i}\right\}_{i \in \mathbb{N}}$ of $C_{0}(\mathbb{R})$ with the property that for each $i \in \mathbb{N}$ almost every point from $A_{i}$, under the iteration of $\tau_{1}$, returns to $A_{i}$ only finitely many times. We define

$$
A_{i}=\left\{\omega \in C_{0}(\mathbb{R}): \omega(t)<0 \quad \forall t:|t| \geq i\right\} .
$$

It follows from Eq. 21 that for $v$-almost every $\omega \in C_{0}(\mathbb{R})$ we have $\lim _{t \rightarrow \infty} \omega(t)=$ $-\infty$ and thus $\cup_{i \in \mathbb{N}} A_{i}=C_{0}(\mathbb{R}) \bmod v$. If $\omega \in A_{i}$, then $\tau_{j} \omega$ is not in $A_{i}$ for $j>i$ since $\tau_{j} \omega(-j)=\omega(0)-\omega(j)=-\omega(j)>0$. Thus, every point from $A_{i}$ returns to $A_{i}$ only finitely many times. This proves the theorem.

Using Theorem 6 we obtain the following corollary, proved in Kabluchko et al. (2009, Theorem 14).

Corollary 2 If $\lim _{t \rightarrow \infty}\left(W_{t}-\sigma_{t}^{2} / 2\right)=-\infty$ a.s., then the corresponding BrownResnick process $\eta^{\vee}$ has a mixed moving maxima representation.

Theorem 12 If $\gamma_{t}$ is bounded, then the corresponding Brown-Resnick process $\eta^{\vee}$ is generated by a positive recurrent flow and, consequently, is non-ergodic. 
Proof To prove the theorem we construct a flow $\left\{\tau_{t}^{\prime}\right\}_{t \in \mathbb{R}}$ which generates $\eta^{\vee}$ (this flow is different from the flow $\left\{\tau_{t}\right\}_{t \in \mathbb{R}}$ constructed in the proof of Theorem 10, but, of course, both flows must be isomorphic by Theorem 2). It is known that if $\gamma_{t}$ is bounded, then it is a variogram of a stationary Gaussian process. Thus, since the law of $\eta^{\vee}$ depends only on $\gamma_{t}$ (and not on $W$ ) by Corollary 1 , we may assume that $W$ is stationary. We construct a spectral representation of $\eta^{\vee}$ as follows. Let $C(\mathbb{R})$ be the space of continuous functions on $\mathbb{R}$, endowed with the product $\sigma$-algebra $\mathcal{B}$. Let the probability measure $v$ be the law of the process $W$ on $C(\mathbb{R})$. Define the non-singular (in fact, measure preserving) flow $\left\{\tau_{t}^{\prime}\right\}_{t \in \mathbb{R}}$ on $(C(\mathbb{R}), \mathcal{B}, v)$ by

$$
\left(\tau_{t}^{\prime} \omega\right)(s)=\omega(s+t) .
$$

Let $f_{0}: C(\mathbb{R}) \rightarrow \mathbb{R}$ be defined by $f_{0}(\omega)=\exp \left(\omega(0)-\sigma_{0}^{2} / 2\right)$. Then

$$
U_{t}^{\prime} f_{0}(\omega)=f_{0}\left(\tau_{t}^{\prime}(\omega)\right)=\exp \left(\omega(t)-\sigma_{0}^{2} / 2\right)=\exp \left(\omega(t)-\sigma_{t}^{2} / 2\right) .
$$

Thus, the pair $\left(\left\{\tau_{t}^{\prime}\right\}_{t \in \mathbb{R}}, f_{0}\right)$ defines a spectral representation of $\eta^{\vee}$ in the sense of Definition 4. Since the flow $\left\{\tau_{t}^{\prime}\right\}_{t \in \mathbb{R}}$ is measure preserving, and since the measure $v$ is finite, it follows that $\left\{\tau_{t}^{\prime}\right\}_{t \in \mathbb{R}}$ is positive recurrent, see Krengel (1985, Theorem 4.6 on p.141). This proves the theorem.

Remark 3 The above Theorems 11 and 12 do not answer completely, by what kind of flow a Brown-Resnick process with given variogram is generated. For example, we do not know, whether there exist a Brown-Resnick process generated by a null-recurrent flow. We also do not know whether the following converse to Theorem 12 holds: if $\gamma_{t}$ is unbounded, then the corresponding Brown-Resnick process has no positive recurrent component in its spectral representation. Another open question is whether it is possible to construct a Brown-Resnick process which is generated by a flow of "mixed" type (e.g. with both dissipative and conservative components non-vanishing).

Remark 4 It is possible to define $\mathrm{S} \alpha \mathrm{S}$ counterparts of Brown-Resnick processes. To this end, one may replace the extremal stochastic integral in Eq. 18 by the $\mathrm{S} \alpha \mathrm{S}$ stochastic integral, that is one may define

$$
\eta^{+}(t)=\int_{\Omega}^{+} \exp \left(\xi_{t}\right) d M_{\mu}^{1} .
$$

The resulting process $\eta^{+}$is stationary, with S1S (Cauchy) marginal distributions. The class of processes constructed in this way is parametrized by negative definite functions (variograms) and thus is somewhat similar to the well-known classes of sub-Gaussian and harmonizable $\mathrm{S} \alpha \mathrm{S}$ processes which are parametrized by positive definite functions. However, whereas both subGaussian and harmonizable processes are non-ergodic by Cambanis et al. (1987), the S1S Brown-Resnick processes, depending on the variogram $\gamma$, can be both positive recurrent (and thus, non-ergodic) and dissipative (and thus, mixing). To see this, combine Theorems 12 and 11 with Theorem 9. 
Acknowledgements I'm grateful to M. Schlather for numerous conversations on max-stable processes and for useful comments on the preliminary version of the paper. I would like also to thank two unknown referees for a very careful reading of the paper and for making a lot of useful suggestions. The author was supported by DFG FOR916.

Open Access This article is distributed under the terms of the Creative Commons Attribution Noncommercial License which permits any noncommercial use, distribution, and reproduction in any medium, provided the original author(s) and source are credited.

\section{References}

Billingsley, P.: Convergence of Probability Measures, 2nd edn. Wiley Series in Probability and Statistics. Wiley, Chichester (1999)

Bretagnolle, J., Dacunha-Castelle, D., Krivine, J.: Lois stables et espaces $L^{p}$. Ann. Inst. Henri Poincaré, Nouv Sér, Sect. B 2 2, 231-259 (1966)

Brown, B., Resnick, S.: Extreme values of independent stochastic processes. J. Appl. Probab. 14, 732-739 (1977)

Cambanis, S., Hardin, C., Weron, A.: Ergodic properties of stationary stable processes. Stoch. Process. their Appl. 24,1-18 (1987)

Gross, A.: Some mixing conditions for stationary symmetric stable stochastic processes. Stoch. Process. their Appl. 51(2), 277-295 (1994)

Gross, A., Robertson, J.: Ergodic properties of random measures on stationary sequences of sets. Stoch. Process. their Appl. 46(2), 249-265 (1993)

de Haan, L.: A spectral representation for max-stable processes. Ann. Probab. 12, 1194-1204 (1984)

de Haan, L., Pickands, J.: Stationary min-stable stochastic processes. Probab. Theory Relat. Fields 72, 477-492 (1986)

Hardin, C.: On the spectral representation of symmetric stable processes. J. Multivar. Anal. 12, 385-401 (1982)

Kabluchko, Z., Schlather, M., de Haan, L.: Stationary max-stable fields associated to negative definite functions. Ann. Probab. www.arXiv:0806.2780v2 [math.PR] (2009, in press)

Krengel, U.: Darstellungssätze für Strömungen und Halbströmungen. I. Math. Ann. 176, 181-190 (1968)

Krengel, U.: Ergodic Theorems. With a Supplement by Antoine Brunel. De Gruyter Studies in Mathematics, 6. Walter de Gruyter, Berlin (1985)

Resnick, S.: Extreme Values, Regular Variation, and Point Processes. Applied Probability, vol. 4. Springer, New York (1987)

Rosinski, J.: Uniqueness of spectral representations of skewed stable processes and stationarity. In: Kunita, H., et al. (eds.) Stochastic Analysis on Infinite Dimensional Spaces. Pitman Research Notes in Mathematics Series, vol. 310, pp. 264-273. Longman Scientific Technical, Harlow (1994)

Rosinski, J.: On the structure of stationary stable processes. Ann. Probab. 23(3), 1163-1187 (1995)

Rosinski, J., Samorodnitsky, G.: Classes of mixing stable processes. Bernoulli 2(4), 365-377 (1996)

de la Rue, T.: Espaces de Lebesgue. In: Azéma, J., et al. (eds.) Séminaire de probabilités XXVII. Lect. Notes Math., vol. 1557, pp. 15-21. Springer, Berlin (1993)

Samorodnitsky, G.: Null flows, positive flows and the structure of stationary symmetric stable processes. Ann. Probab. 33(5), 1781-1803 (2005)

Samorodnitsky, G., Taqqu, M.: Stable Non-Gaussian Random Processes: Stochastic Models with Infinite Variance. Stochastic Modeling. Chapman Hall, New York (1994)

Schlather, M.: Models for stationary max-stable random fields. Extremes 5(1), 33-44 (2002)

Schreiber, M.: Quelques remarques sur les caractérisations des espaces $L^{p}, 0 \leq p<1$. Ann. Inst. Henri Poincaré, Nouv Sér, Sect. B 2 8, 83-92 (1972)

Stoev, S.: On the ergodicity and mixing of max-stable processes. Stoch. Process. their Appl. 118, 1679-1705 (2008)

Stoev, S., Taqqu, M.: Extremal stochastic integrals: a parallel between max-stable processes and $\alpha$-stable processes. Extremes 8(4), 237-266 (2005)

Surgailis, D., Rosinski, J., Mandrekar, V., Cambanis, S.: Stable mixed moving averages. Probab. Theory Relat. Fields 97(4), 543-558 (1993) 